\title{
Spectropolarimetry of stars across the H-R diagram
}

\author{
Swetlana Hubrig and Markus Schöller
}

\begin{abstract}
The growing sample of magnetic stars shows a remarkable diversity in the properties of their magnetic fields. The overall goal of current studies is to understand the origin, evolution, and structure of stellar magnetic fields in stars of different mass at different evolutionary stages. In this chapter we discuss recent measurements together with the underlying assumptions in the interpretation of data and the requirements, both observational and theoretical, for obtaining a realistic overview of the role of magnetic fields in various types of stars.
\end{abstract}

\section{Introduction}

The presence of a magnetic field in the Sun was detected in 1908 by Hale and the first detection of a magnetic field in a distant star was achieved in 1947 by Babcock. Since then, a lot of effort has been put into investigations of solar and stellar magnetism to understand how and why so many stars, including our own Sun, are magnetic, and what the implications might be for life on Earth and elsewhere in the universe. The past two decades have seen a significant step forward in our understanding of the occurrence of magnetic fields in stars of different mass and different age. This became possible through the significant progress in spectropolarimetric surveys of surface magnetic fields that made use of the currently available spectropolarimeters ESPaDOnS (Echelle SpectroPolarimetric Device for the Observation of Stars) at the Canada-France-Hawaii telescope, NARVAL at the Bernard Lyot $2 \mathrm{~m}$ Cassegrain telescope, the Main Stellar Spectrograph of the Russian $6 \mathrm{~m}$ tele-

Swetlana Hubrig

Leibniz-Institut für Astrophysik Potsdam (AIP), An der Sternwarte 16, 14482 Potsdam, Germany, email: shubrig@aip.de

Markus Schöller

European Southern Observatory, Karl-Schwarzschild-Str. 2, 85748 Garching, Germany, email: mschoell@eso.org 
scope, FORS 1 and FORS 2 (FOcal Reducer low dispersion Spectrographs) at the ESO Very Large Telescope (VLT), and HARPSpol (High Accuracy Radial velocity Planet Searcher) at ESO/La Silla.

As of today, magnetic fields are found at all stages of stellar evolution, from brown dwarfs and young T Tauri stars to the end products of stellar evolution: white dwarfs and neutron stars. The incidence of magnetic fields in stars is diverse. The presence of a convective envelope is a necessary condition for significant magnetic activity in stars and magnetic activity is found all the way from late A-type stars, e.g. in Altair (Robrade \& Schmitt 2009) with very shallow convective envelopes, down to the coolest fully convective M-type stars. All solar type stars appear to be magnetic, the stronger the more rapidly they rotate (e.g. Pallavicini et al. 1981). This is understood through the $\alpha \Omega$-dynamo, which is thought to operate in the convective envelope of these stars (e.g. Schmitt 1987). In intermediate mass main sequence stars, only about $10 \%$ are found to have kG-strength large-scale magnetic fields. Here, the correlation of magnetic fields with stellar rotation is opposite to that of solar type stars: whereas most intermediate mass stars are rapid rotators throughout their main sequence life, the magnetic stars are mostly slow rotators (e.g., Mathys 2017; Hubrig et al. 2018a).

In spite of the fact that recent years have seen a significant increase in observational studies of stellar magnetism, the most important aspects, such as the origin of stellar magnetic fields and the evolution of magnetic field configurations - the largescale organised magnetic fields observed in massive stars or the complex magnetic topology observed in low-mass stars - are still not understood.

For massive and intermediate mass stars with radiative envelopes, it has been argued that their magnetic fields could be fossil relics of the fields that were present in the interstellar medium from which these stars have formed (e.g. Moss 2003). A search for the presence of magnetic fields in massive stars located in active sites of star formation, in the $\rho$ Ophiuchus star-forming cloud and in the Trifid nebula, led to the detection of magnetic fields of several $\mathrm{kG}$ in two early B-type stars, the B2 V star HD 147933 and the B1 V star HD 164492Cb (Hubrig et al. 2018b, 2014a). However, the fossil field hypothesis has several problems as it does not explain the low $(\sim 10 \%)$ occurrence of magnetic stars and their broad range of field strengths. Moreover, a study of the distribution of magnetic Ap stars in the H-R diagram using accurate Hipparcos parallaxes by Hubrig et al. (2000) showed that these stars are concentrated toward the centre of the main-sequence band and only rarely can be found close to the zero-age main sequence (ZAMS). Similar studies using Gaia DR2 data are on the way, but their results are not published yet. Alternatively, magnetic fields may be generated by strong binary interaction, i.e., in stellar mergers, or during a mass transfer or common envelope evolution (e.g. Tout et al. 2008). The resulting strong differential rotation is considered as a key ingredient for the generation of magnetic fields (Petrovic et al. 2005). For Galactic O-type stars, it was shown that the fraction of very close binaries is so high that about one third of them interacts with a companion during their main sequence evolution through mass transfer or merging (Sana et al. 2012). 
In cool, low-mass stars, the key unresolved aspects are the origin of the solarlike activity cycle, the origin and character of differential rotation and its role in the large-scale dynamo, and the origin of high energy coronal activity. Knowledge of the evolution of the magnetic field and its configuration is extremely important for our understanding of the past and future of the Sun and the solar system. Notably, low-mass stars are complex magnetohydrodynamical objects that require state-ofthe-art observations and numerical simulations to pin down the physical processes that are responsible for the diversity and dynamics of these stars. The origin of stellar activity in low-mass stars resides most likely in the stellar convection zones. The mechanism involves helical gas motions and large-scale differential rotation. The most straightforward combination of these effects is the $\alpha \Omega$-dynamo, which can reproduce many basic properties of the solar activity cycle. Differential rotation generates large-scale toroidal fields by winding up the poloidal field. Differential rotation is the main field generator in the $\alpha \Omega$-dynamo, which therefore maintains predominantly axisymmetric fields that are dominated by the toroidal component. On the other hand, differential rotation alone is not sufficient for dynamo action, as it can not prevent the decay of the poloidal field. The $\alpha$-effect, which is caused by the Coriolis force, is vital to the dynamo, because it produces a poloidal field from the toroidal field, thus completing the field-amplifying dynamo process. In contrast to differential rotation, the $\alpha$-effect can maintain a magnetic field by itself. This type of dynamo is known as the $\alpha^{2}$-dynamo. Unlike the $\alpha \Omega$-dynamo, it prefers non-axisymmetric field configurations that are not dominated by one component. Mean field magnetohydrodynamics provides a very successful theoretical model for stellar differential rotation and the accompanying meridional flows (Kitchatinov \& Rüdiger 1999; Küker \& Stix 2001).

In the following sections, we discuss magnetic field diagnostics, observations of magnetic fields in upper main-sequence, pre-main-sequence, and low-mass active stars, finishing with post-main-sequence stars and degenerate stellar remnants.

\section{Magnetic field diagnostics}

The currently applied observing techniques differ for different types of stars, involving the Zeeman effect, the Paschen-Back effect, and the molecular Zeeman effect. Observations of broad-band linear polarization, which is caused by different saturation levels of the $\pi$ and $\sigma$ components of a spectral line in the presence of a magnetic field, were reported in the past for a few chemically peculiar magnetic Ap stars (e.g. Leroy 1989; Landolfi et al. 1993). This differential effect for Zeeman components is qualitatively similar for all lines, so that in broad-band observations the contributions of all lines add up. Monitoring of broad-band linear polarization of a few Ap stars over a few years was used to establish the presence of their extremely slow rotation. Studies of broad-band linear polarization do not represent a widely used technique of stellar magnetic field diagnosis, and nowadays they are superseded by the observations of linear polarization in individual spectral lines. 
The most complete way to study stellar magnetic fields is to observe both linear and circular polarization, i.e. all four Stokes parameters $I, Q, U$, and $V$. However, such observations are very challenging as in disk-integrated observations the signal can be canceled or significantly decreased due to mixed polarity fields. Furthermore, measurements of linear polarization are sensitive to instrumental polarization. Therefore, only large-scale magnetic fields can be studied using the full Stokes vector. Importantly, physically realistic models of the geometric structure of magnetic fields in stellar atmospheres can be achieved only if linear polarisation measurements are implemented.

Observing the spectrum of a star in the two senses of circular polarisation is in almost all cases the most sensitive method of detecting weak magnetic fields on a stellar surface. The measurement of the Stokes $V$ parameter permits to detect the mean longitudinal magnetic field, which is a line intensity weighted average over the visible hemisphere of a star of the magnetic field component directed along the lineof-sight. To measure longitudinal magnetic fields, in particular observing with lowresolution spectropolarimeters, a regression analysis approach is frequently used (e.g. Bagnulo et al. 2001). The mean longitudinal magnetic field measurement $\left\langle B_{\mathrm{Z}}\right\rangle$ in this approach is based on the relation

$$
\frac{V}{I}=-\frac{g_{\text {eff }} e \lambda^{2}}{4 \pi m_{\mathrm{e}} c^{2}} \frac{1}{I} \frac{\mathrm{d} I}{\mathrm{~d} \lambda}\left\langle B_{\mathrm{z}}\right\rangle
$$

where $V$ is the Stokes parameter that measures the circular polarization, $I$ is the intensity in the unpolarized spectrum, $g_{\text {eff }}$ is the effective Landé factor, $e$ is the electron charge, $\lambda$ is the wavelength, $m_{\mathrm{e}}$ is the electron mass, $c$ is the speed of light, $\mathrm{d} I / \mathrm{d} \lambda$ is the wavelength derivative of Stokes $I$, and $\left\langle B_{\mathrm{Z}}\right\rangle$ is the mean longitudinal magnetic field. Although the longitudinal magnetic field measurements present the canonical diagnostics of stellar magnetic fields, other observational and analytical methods to diagnose the presence and structure of magnetic fields in stellar atmospheres are frequently applied.

In a number of $A p$ and Bp stars whose projected rotational velocity is sufficiently small and whose magnetic field is strong enough to exceed the rotational Doppler broadening, certain lines with suitable Zeeman patterns show resolved Zeeman split lines in unpolarized light (Preston 1971; Mathys 1990). They are used to estimate the line-intensity weighted average of the modulus of the magnetic field over the visible stellar hemisphere, the mean magnetic field modulus. Simultaneous knowledge of the mean magnetic field modulus and the longitudinal magnetic field over the stellar rotation period permits to set constraints on the magnetic field geometry.

Since the Zeeman signatures in the spectra of many stars are generally very small, and increasing the signal-to-noise ratio $(S / N)$ by increasing the exposure time is frequently limited by the short rotation period of the star, multi-line approaches as proposed by Semel (1989) are commonly used to increase the $S / N$. The most widely used approach is the Least Squares Deconvolution (LSD; Donati et al. 1997). The main assumption in LSD is the application of the weak field approximation, that is, the magnetic splitting of spectral lines is assumed to be smaller than their Doppler 
broadening. Furthermore, it is assumed that the local line profiles are self-similar and can be combined into an average profile. Due to non-linear effects in the summation and the effect of blends, the resulting LSD profiles should not be considered as observed, but rather processed Zeeman signatures. In a number of recent studies, a novel magnetometric technique, the multi-line Singular Value Decomposition (SVD) method was applied. The software package to study weak magnetic fields using the SVD method was introduced by Carroll et al. (2012). The basic idea of SVD is similar to the principal component analysis (PCA) approach, where the similarity of individual Stokes $V$ profiles permits to describe the most coherent and systematic features present in all spectral line profiles as a projection onto a small number of eigenprofiles.

Other popular methods to exploit the information contained in polarised spectral line profiles currently include the moment technique, in particular the determination of the crossover effect and of the mean quadratic field (e.g. Mathys 1993), and Zeeman-Doppler imaging (ZDI). In the moment technique, the shapes of the spectral lines observed in Stokes $I$ and Stokes $V$ profiles can be characterized by their moments of various orders about the line centre and can be interpreted in terms of quantities related to the magnetic field. The analysis is usually based on the consideration of samples of reasonably unblended lines and critically depends on the number of lines that can be employed. ZDI is an inversion technique applied to time-series of two or four Stokes parameters, introduced by Semel (1989). It allows to recover the distribution of the temperature (on the surface of cool stars) or of chemical spots (on the surface of Ap and Bp stars) and of the magnetic field vector over the stellar surface. In most studies, however, only Stokes $I$ and Stokes $V$ observations are used to obtain Zeeman-Doppler stellar images. Obviously, in such cases, the solution is not unique and is constrained by a number of assumptions. In the studies of cool stars with temperature spots it is however possible to remedy this situation by the implementation of full polarized radiative transfer in both atomic and molecular lines. Employing molecular lines is essential to localize magnetic fields inside cool regions on the stellar surface (e.g. Sennhauser et al. 2009).

A novel technique to detect surface magnetic fields in A- and F-type stars, which uses the autocorrelation of spectra in integral light, was recently suggested by Borra $\&$ Deschatelets (2015). The original idea of the autocorrelation technique is to measure the width of spectral lines and to disentangle the magnetic broadening from other broadening effects, using the differences in wavelength dependence. Such a technique can be applied to low-resolution spectra, down to stars that are too faint for high-resolution observations even with large telescopes. The availability of this technique allows one to use publicly available data archives from numerous spectrographs all over the world to obtain reliable statistics on the occurrence of magnetic fields in A and F stars in clusters and the field at different evolutionary stages. Using this technique, it will become possible even to search for magnetic fields in a sample of early B-type stars in the Magellanic Clouds using low-resolution spectroscopic observations. 


\section{O- and early B-type stars}

Massive stars with initial masses ranging from 8 to $100 M_{\odot}$ are of particular interest, as they end their evolution with a supernova explosion, producing neutron stars or black holes. Magnetic O-type stars with masses larger than $30 M_{\odot}$ and their WR descendants have been suggested as progenitors of magnetars (Gaensler et al. 2005). A magnetic mechanism for the collimated explosion of massive stars, relevant for long-duration gamma-ray bursts, X-ray flashes, and asymmetric core collapse supernovae was proposed by e.g. Uzdensky \& MacFadyen (2006).

Recent observations indicate that probably only a small fraction of about $7 \pm 3 \%$ of O-type stars with masses exceeding $18 M_{\odot}$ (Grunhut et al. 2017) and about $6 \pm 3 \%$ of early B-type and O-type stars (Schöller et al. 2017) possess measurable, mostly dipolar magnetic fields. Progress in the studies of massive stars became possible mostly through the MiMeS (The Magnetism in Massive Stars, Wade et al. 2011), MAGORI (MAGnetic field ORIgin, Hubrig et al. 2011), and BOB (B-fields in OB stars, Morel et al. 2015) surveys, which made use of the spectropolarimeters ESPaDOnS, NARVAL, FORS2, and HARPSpol. The vast majority of magnetic massive stars exhibit a smooth, single-wave variation of the longitudinal magnetic field during the stellar rotation cycle. These observations are considered as evidence for a dominant dipolar contribution to the magnetic field topology.

Braithwaite \& Spruit (2004) showed that stars with radiative envelopes can host stable large-scale magnetic field configurations, as those observed in the Ap/Bp stars, independent from the star's rotation. The implication is that up to about 30$50 \%$ of all massive stars might be magnetic, and that so far only the tip of the iceberg has been seen (Stello et al. 2016). From Kepler and Kepler K2 photometry, Balona (2013, 2016) concluded that about $40 \%$ of A-type stars and $30-50 \%$ of late $\mathrm{O}$ and B-type stars show rotational modulation due to the presence of star spots. Pulsating stars, eclipsing binaries, and ellipsoidal variables of high amplitude were avoided in these studies. Since it is difficult to conceive of starspot formation without the presence of a magnetic field, these results indicate that the occurrence of magnetic fields in A, B, and late O-type stars is greatly underestimated.

No complete volume-limited study of the incidence of magnetism in massive stars has been presented so far. Thus one of the major goals of recent studies is to build trustworthy statistics on the occurrence of magnetic fields in massive stars and their topology at different evolutionary stages. This is critical to answer the principal question of the possible origin of such fields. Previously observed samples greatly suffer from a number of biases in the target selection related to specific spectral classifications. We know already that all five Galactic stars with Of?p classification are magnetic and at least $10 \%$ of the early-type B stars belong to the magnetic Herich group, containing stars with overabundances of Si and He. O-type stars with Of?p classification exhibit recurrent, and apparently periodic, spectral variations in Balmer, He I, C III, and Si III lines, sharp emission or P Cygni profiles in He I and the Balmer lines, and strong C III emission around $4650 \AA$ (Walborn 1972). A search for the presence of magnetic fields at different evolutionary stages is also predominantly carried out in bright stars that are slow rotators. Since very young 
massive stars at the ZAMS are usually faint, this evolutionary stage of massive stars remains completely unexplored. The results recently presented in several papers reflect the lack of observations of young massive stars close to the ZAMS (e.g. Castro et al. 2015).

It is quite possible that a significant fraction of the magnetic massive stars remains undiscovered due to a more complex magnetic field topology related to the presence of a subsurface convection zone. Multi-wavelength observations of massive stars show cyclical line profile structures, the discrete absorption components (DACs). Their presence indicates that stellar prominences can emerge on the stellar surface, probably due to localised magnetic activity, more or less in analogy to what is observed on the solar surface (e.g. Sudnik \& Henrichs 2016). Action of a subsurface convection zone (Cantiello et al. 2009) would be the most likely driving mechanism that generates magnetic spots, which are the source of those prominences. This zone may also be the source of a global magnetic field, winding up toroidally with stochastic buoyancy breakouts, causing corotating magnetic bright spots at the surface of the star (Cantiello \& Braithwaite 2011).

Atmospheric magnetic fields can impact surface rotation rates via magnetic braking (Weber \& Davis 1967; ud-Doula et al. 2008), introduce chemical abundance inhomogeneities and peculiarities (Hunger \& Groote 1999), and confine the stellar wind in a magnetosphere (e.g., Friend \& MacGregor 1984; ud-Doula \& Owocki 2002). The ejected matter streams along the field lines towards the magnetic equator and collides in the vicinity of the equatorial plane, leading to strong shocks and thereby heating plasma to X-ray temperatures (Babel \& Montmerle 1997).

The strongest longitudinal magnetic field in an $\mathrm{O}$ star of more than $5 \mathrm{kG}$ was detected in the Of?p star NGC 1624-2, suggesting a dipole component of about $20 \mathrm{kG}$ (Wade et al. 2012). Among the early B-type stars, the record holder is the He-rich star CPD $-62^{\circ} 2124$ with a dipole component of at least $21 \mathrm{kG}$ (Hubrig et al. 2017).

Noteworthy, the survey of the BOB consortium, aiming to establish a lower boundary of magnetic fields in massive stars, revealed the presence of very weak Zeeman signatures across the line profiles in high-quality HARPSpol spectra of two early B-type stars. This work indicated that also magnetic fields of the order of a few Gauss can be present in massive stars, but possibly escape their detection due to insufficient measurement accuracy (e.g. Fossati et al. 2015).

Wolf-Rayet (WR) stars form an integral part of the late evolution of the most massive stars. While the WR core He-burning phase lasts only $\sim 10 \%$ of the O-star main sequence phase, WR winds are at least ten times stronger than on the main sequence. With wind-momenta in WR stars reaching up to 30 times the available photon momentum, magnetic fields have been proposed as additional or alternative wind-driving mechanisms to radiation pressure (Cassinelli et al. 1995). The detection of magnetic fields in WR stars is however difficult, chiefly because the line spectrum is formed in the strong stellar wind. This does not only imply a dilution of the field at the place of line formation. The biggest problem is the wind broadening of the emission lines by Doppler shifts with wind velocities of a few thousand $\mathrm{km} \mathrm{s}^{-1}$. de la Chevrotiére et al. (2014) used ESPaDOnS spectra to search for magnetic fields in eleven WR stars. They reported that the data show evidence 
supporting marginal detections of a magnetic field of the order of a few hundred Gauss and less in three WR stars. A search for magnetic fields in several WR stars using low-resolution FORS 2 spectropolarimetry showed the presence of a magnetic field in the cyclically variable star WR 6 at a significance level of $3.3 \sigma(258 \pm 78 \mathrm{G})$. In this work, a theoretical approach to estimate the magnetic field strength in stars with strong emission lines was considered for the first time (Hubrig et al. 2016).

\section{Ap and Bp stars}

Globally ordered magnetic fields are observed in roughly $10 \%$ of the intermediate and massive main-sequence stars with spectral types between approximately B2 and F0. These stars, generally called chemically peculiar Ap and Bp stars, exhibit strong overabundances of certain elements, such as iron peak elements and rare earths, and underabundances of $\mathrm{He}, \mathrm{C}$, and $\mathrm{O}$, relative to solar abundances, and are characterized observationally by anomalous line strengths. Massive Bp stars usually show overabundances of $\mathrm{He}$ and $\mathrm{Si}$. As the star rotates, the magnetic field and surface abundance distribution is observed from various aspects, resulting in variability of the measured magnetic field and spectral line strengths.

The variable magnetic fields are generally diagnosed through mean longitudinal magnetic field, mean magnetic field modulus, and net broadband linear polarization measurements. Chemical abundance anomalies are commonly believed to be due to radiatively driven microscopic diffusion in stars rotating sufficiently slowly to allow such a process to be effective (e.g. Michaud 1970). While two thirds of all Galactic O-type stars appear to be members of close binary systems (Sana et al. 2012), the study of Carrier et al. (2002) indicated a dearth of magnetic Ap stars stars in close binaries. Only for two close binary systems with Ap magnetic components, HD 98088 (e.g., Babcock 1958; Abt et al. 1968) and HD 161701 (Hubrig et al. 2014b), is the structure of the magnetic field known. Remarkably, in both systems carries the surface of the Ap component, which is facing the companion, a positive magnetic field. It is, however, not clear whether tidal forces may play a role during the dynamic process of tidal synchronisation, to align the field geometries in the observed way. Further systematic searches for magnetic fields in binary systems should be conducted to properly evaluate theoretical models of the origin of their magnetic fields.

Currently, 84 stars with magnetically split components are known and their study permits to establish the general properties of their magnetic fields (e.g. Mathys 2017). The star with the strongest magnetic field currently known, "Babcock's star" HD 215441 is a B8 V star with a surface dipole magnetic field strength of $34 \mathrm{kG}$ (Babcock 1960; Preston 1969). Zeeman split lines were recently discovered in a few early Bp stars with low projected rotational velocity and strong, $\mathrm{kG}$ order magnetic fields (Hubrig et al., in prep.). Examples of magnetically split lines in three such stars are presented in Fig. 1. 

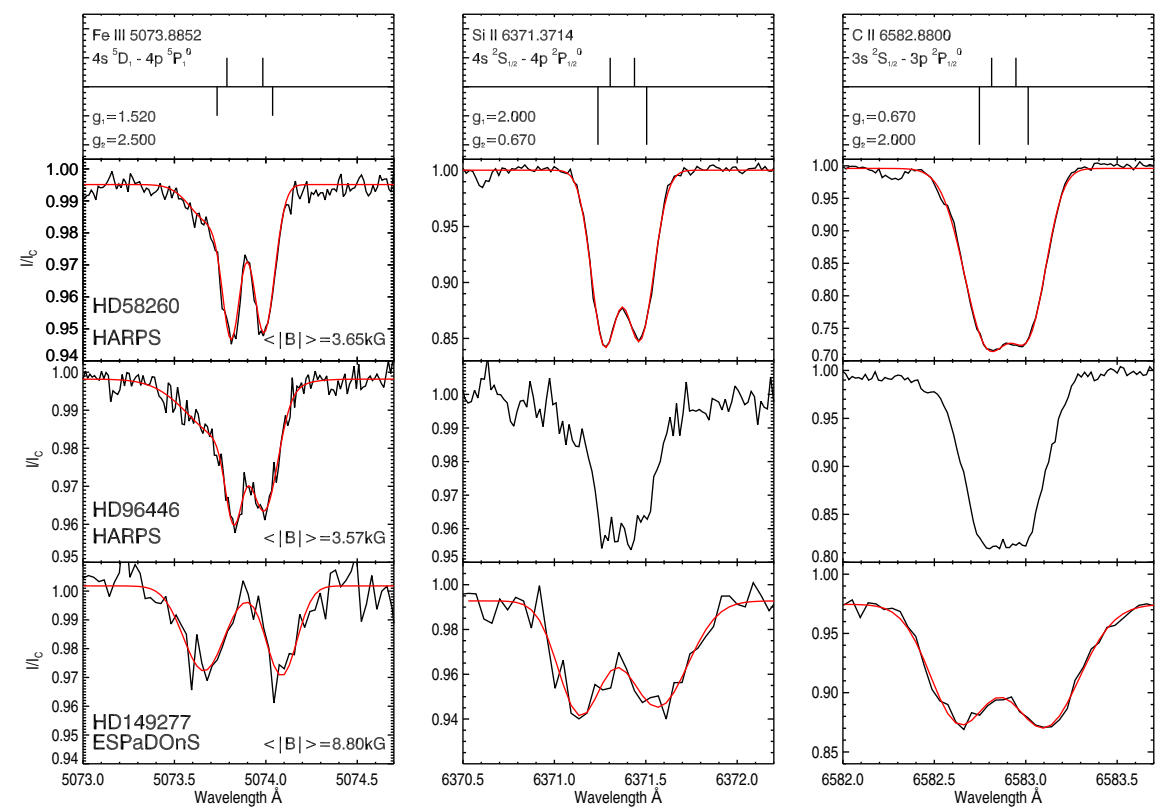

Fig. 1 The magnetically split lines Fe III $\lambda$ 5074, Si II $\lambda 6371$, and C II $\lambda 6583$ in high-resolution Stokes $I$ spectra of the early B-type stars HD 58260, HD 96446, and HD 149277. The red lines denote the fit of a multi-Gaussian to the data. For two lines in the spectrum of HD 96446, the splitting is not sufficient to allow a proper fit.

The evolution of magnetic field configurations in stars with masses below $6 M_{\odot}$ using a sample of Ap and Bp stars with accurate Hipparcos parallaxes and definitely determined longitudinal magnetic fields, was until now considered only in the study by Hubrig et al. (2007). This work showed that stronger magnetic fields are detected in younger stars. No evidence was detected for any loss of angular momentum during the main-sequence life. Also the magnetic flux was found to be constant over the stellar life time on the main sequence. An excess of stars with large obliquities $\beta$ (the orientation of the magnetic axis with respect to the rotation axis) was detected in both higher and lower mass stars. The evolution of $\beta$ appeared to be somewhat different for stars with $\mathrm{M}<3 M_{\odot}$ than for stars with $\mathrm{M}>3 M_{\odot}$, for which a strong hint for an increase of $\beta$ with time spent on the main sequence was discovered.

Using the indirect surface mapping ZDI method is a valuable option to derive magnetic field maps, but was applied only for a few Ap/Bp stars during the last years. A summary of ZDI results is presented in the work of Kochukhov et al. (2018). The authors find that the global magnetic field geometry changes little from one star to another, with nearly all stars showing dominant dipolar fields with a varying degree of distortion. 


\section{Pre-main sequence stars}

Studies of magnetic fields in stars at early evolutionary stages, before they arrive on the main sequence, are of special interest to get an insight into the magnetic field origin. Moreover, such studies are extremely important because they enable us to improve our insight into how the magnetic fields in these stars are generated and how they interact with their environment, including their impact on the planet formation process and the planet-disk interaction.

It is generally accepted that magnetic fields are important ingredients of the star formation process (e.g. McKee \& Ostriker 2007) and are already present in stars in the pre-main sequence (PMS) phase. The PMS low mass T Tauri stars stand out by their very large emissions in chromospheric and transition-region lines. The accretion of circumstellar disk material onto the surface of these stars is controlled by a strong stellar magnetic field. These stars are usually grouped into two classifications: classical T Tauri stars, which show evidence of a circumstellar disk and mass accretion onto the central star in the form of excess emission in X-rays, the UV, optical, and infrared, and weak-line T Tauri stars, which do not show evidence for significant mass accretion. A model for the generation of magnetic fields in fully convective PMS stars was presented by e.g., Küker \& Rüdiger (1999), Chabrier \& Küker (2006), and Küker (2009), who considered a dynamo process of $\alpha^{2}$-type.

The accretion of circumstellar disk material onto the surface of T Tauri stars is assumed to be controlled by a strong stellar magnetic field. Furthermore, the magnetic field appears to be critical for explaining their rotational properties (e.g. Herbst et al. 2007) and probably plays a critical role in driving the observed outflows (Mohanty \& Shu 2008). Magnetic field measurements of these stars over the last decades show the presence of $\mathrm{kG}$ magnetic fields. Basri et al. (1992) were the first to detect a magnetic field on the surface of a T Tauri star using Zeeman broadening of magnetically sensitive lines in Stokes $I$. Modeling Zeeman-broadened Stokes $I$ profiles often involves template spectra of magnetically active and inactive stars, which are weighted by a filling factor. As this method is sensitive to the magnetic field distribution but has a limited sensitivity to the magnetic field geometry, most recent studies involve circular polarization observations to characterize surface magnetic fields using the ZDI technique. On the other hand, according to Carroll et al. (2012), a common problem for ZDI is that the real strong magnetic fields that are associated with cool surface regions produce only a fraction of the photon flux compared to the unaffected quiet or even hot surface regions. When studying the correlation between the magnetic field and temperature spots, it is important to be aware that the detectability of the field is weighted by the surface brightness. To prevent such an ill-defined flux-weighting and the mutual effects between the temperature and magnetic field, Carroll et al. have pursued a strategy that performs a simultaneous DI and ZDI to retrieve both the temperature and the magnetic field distribution at the same time. Their reconstruction of the temperature and magnetic field of the weakline T Tauri star V410 Tau revealed that the majority of the strong fields of $2 \mathrm{kG}$ are located in cool spots, in particular within the large polar spot. The reconstruction clearly showed that both polarities coexist within the large polar spot and that the 
entire polar-field topology appears to be dominated by a twisted bi-polar structure separated by a relatively sharp neutral line.

To study magnetic field topologies in T Tauri stars, the group of Donati (e.g. Donati et al. 2015) applies a stellar-surface tomographic-imaging package. This imaging code is set up to invert (both automatically and simultaneously) time series of Stokes $I$ and $V$ LSD profiles into brightness and magnetic maps of the stellar surface, where brightness imaging is allowed to reconstruct both cool spots and warm plages, known to contribute to the activity of very active stars. This tool is based on the principles of maximum-entropy image reconstruction and on the assumption that the observed variability is mainly caused by rotational modulation with an added option for differential rotation.

According to Adams \& Gregory (2012), in accreting systems, the polar strength of the dipole component is particularly relevant for determining the disk truncation radius and the balance of torques in the star-disk system. Johnstone et al. (2014) found that the dipole strength is correlated with the rotation period: stars with weak dipole components tend to be rotating faster than stars with strong dipole components. Recent observations of magnetic fields in a small sample of T Tauri stars suggest that the magnetic field topology changes as a function of age, i.e. there is a relation between the large-scale magnetic field and the position in the H-R diagram. Gregory et al. (2012) reported that the magnetic field gains complexity with the evolution of the star: the magnetic field is mainly dipolar when the star is completely convective. After the development of the radiative core the dipolar component looses power compared to high order components of the multipole expansion. Based on magnetohydrodynamic simulations, Zaire et al. (2017) suggest that the presence of a tachocline could be an important factor in the development of magnetic fields with higher multipolar modes.

The presence of magnetic fields in higher mass PMS stars, the Herbig Ae/Be stars, has long been suspected, in particular on account of $\mathrm{H} \alpha$ spectropolarimetric observations pointing out the possibility of magnetospheric accretion, similar to that of classical T Tauri stars (e.g. Vink et al. 2002). While models of magnetically driven accretion and outflows successfully reproduce many observational properties of the classical T Tauri stars, the picture is completely unclear for the Herbig Ae/Be stars, mostly due to the poor knowledge of their magnetic field topology. So far, the magnetic field geometry was constrained only for two Herbig Ae/Be stars, V380 Ori (Alecian et al. 2009) and HD 101412 (Hubrig et al. 2011), and only about 20 Herbig stars were reported to host magnetic fields (Hubrig et al. 2015, and references therein).

The two best studied Herbig Ae/Be stars exhibit a single-wave variation in their mean longitudinal magnetic field during the stellar rotation cycle. This behaviour is usually considered as evidence for a dominant dipolar contribution to the magnetic field topology. Presently, the Herbig Ae star HD 101412 possesses the strongest magnetic field ever measured in any Herbig Ae star, with a surface magnetic field $\langle B\rangle$ of up to $3.5 \mathrm{kG}$. HD 101412 is also the only Herbig Ae/Be star for which the rotational Doppler effect was found to be small in comparison to the magnetic split- 
ting, presenting several spectral lines resolved into magnetically split components observed in unpolarised light at high spectral resolution (Hubrig et al. 2010).

Notably, the task of magnetic field measurements in Herbig stars is very challenging, as the work of Hubrig et al. (2015) demonstrates, in which the authors compiled all magnetic field measurements reported in previous spectropolarimetric studies. This study indicates that the low detection rate of magnetic fields in Herbig Ae stars, about 7\% (Alecian et al. 2013), can indeed be explained not only by the limited sensitivity of the published measurements, but also by the weakness of these fields. The obtained density distribution of the rms longitudinal magnetic field values reveals that only a few stars have magnetic fields stronger than $200 \mathrm{G}$, and half of the sample possesses magnetic fields of about $100 \mathrm{G}$ and less. Consequently, the currently largest spectropolarimetric survey of magnetic fields in several tens of Herbig stars by Alecian et al. (2013) using ESPaDOnS and NARVAL cannot be considered as representative: the measurement accuracy in this study is worse than $200 \mathrm{G}$ for $35 \%$ of the measurements, and for $32 \%$ of the measurements it is between 100 and $200 \mathrm{G}$. Clearly, to improve our understanding of the origin of magnetic fields in Herbig Ae/Be stars and their interaction with the protoplanetary disk, it is of utmost importance to study magnetic fields with high accuracy measurements in a representative sample of Herbig Ae/Be stars.

Zeeman signatures in the spectra of Herbig Ae/Be stars are generally very small, and increasing the $S / N$ by increasing the exposure time is frequently limited by the short rotation period of the star. Therefore, multi-line approaches such as LSD and SVD are commonly used to increase the $S / N$. An example of a very weak Zeeman feature in the HARPSpol spectra of the Herbig Ae star PDS 2 detected using the SVD method (Hubrig et al. 2015) is presented in Fig. 2.

Spectropolarimetric observations of a sample of 21 Herbig Ae/Be stars observed with FORS 1 were used to search for a link between the presence of a magnetic field and other stellar properties by Hubrig et al. (2009). This study did not indicate any correlation of the strength of the longitudinal magnetic field with disk orientation, disk geometry, or the presence of a companion. No simple dependence on the massaccretion rate was found, but the range of the observed field values qualitatively supported the expectations from magnetospheric accretion models with dipole-like field geometries. Both the magnetic field strength and the X-ray emission showed a decline with age in the range of $\sim 2-14 \mathrm{Myr}$ probed by the sample, supporting a dynamo mechanism that decays with age. In particular, it was found that magnetic fields are strong in very young Herbig stars and become very weak or completely disappear at the end of their PMS life. In line with these results, Hubrig et al. (2000, 2005, 2007) showed that magnetic fields in Ap stars with masses less than $3 M_{\odot}$ are rarely found close to the ZAMS and that $\mathrm{kG}$ magnetic fields appear in A stars already evolved from the ZAMS. Thus, it is very unlikely that Herbig stars are the progenitors of the magnetic Ap stars. Importantly, the Herbig stars seem to obey the universal power-law relation between magnetic flux and X-ray luminosity established for the Sun and main-sequence active dwarf stars (Pevtsov et al. 2003). Future work on stellar properties of magnetic Herbig stars should involve a larger 


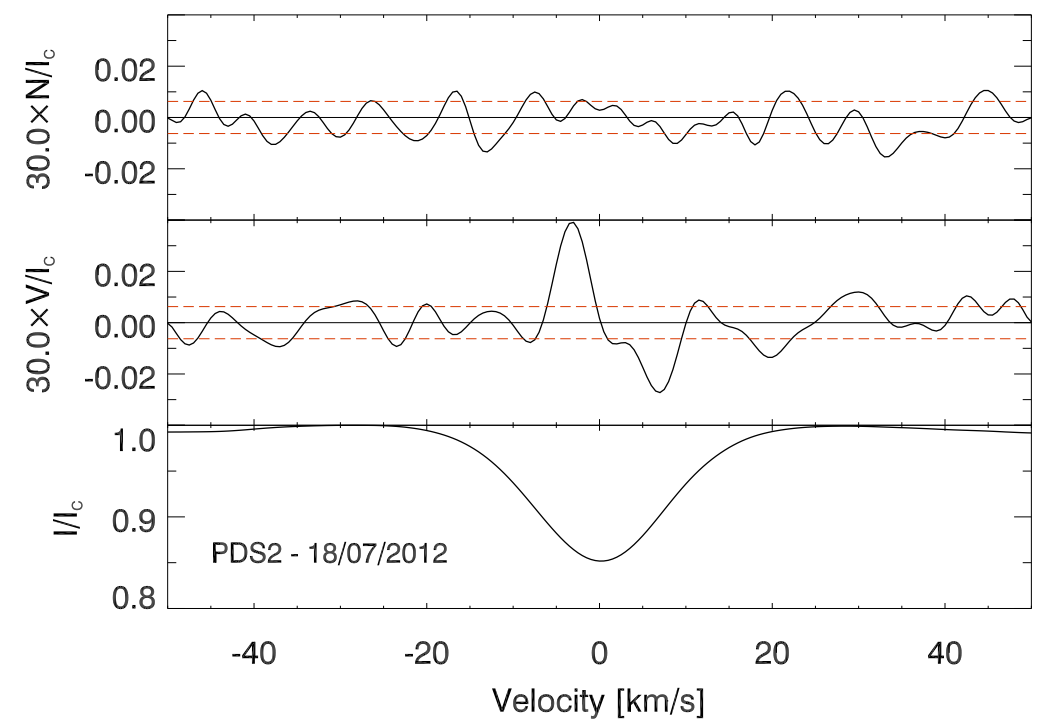

Fig. 2 Detection of a mean longitudinal magnetic field in the Herbig Ae star PDS 2. The Stokes $I$ (bottom), Stokes $V$ (middle), and diagnostic null ( $N$; top) SVD profiles were used to determine $\left\langle B_{z}\right\rangle=33 \pm 5 \mathrm{G}$. The Stokes $V$ and $N$ profiles were expanded by a factor of 30 . The red dashed lines indicate the standard deviations for the Stokes $V$ and $N$ spectra. This figure corresponds to the lower part of Fig. 2 in "The prevalence of weak magnetic fields in Herbig Ae stars: the case of PDS 2" by Hubrig et al. (2015), MNRAS 449, L118.

and more representative sample to determine the existing relations at a higher confidence level.

As already mentioned in Sect. 4, in agreement with the merging scenario for the origin of magnetic fields in magnetic stars with radiative outer zones, the number of close binary systems with Ap components is very low. Similarly, studies of Herbig Ae stars by Wheelwright et al. (2010) and Duchêne (2015) indicate the lack of close binary systems with $\mathrm{P}_{\text {orb }}<20 \mathrm{~d}$. Weak magnetic fields were recently detected in two Herbig Ae systems, in AK Sco and in the presumed binary HD 95881. Using high quality HARPSpol observations, Järvinen et al. (2018) obtained $\left\langle B_{z}\right\rangle=-83 \pm 31 \mathrm{G}$ for the secondary component in the system AK Sco and $\left\langle B_{z}\right\rangle=-93 \pm 25 \mathrm{G}$ for HD 95881. It is of interest that for AK Sco they observe the magnetic field in the secondary component in the region of the stellar surface facing permanently the primary component, meaning that the magnetic field geometry in the secondary component is likely related to the position of the primary component. A similar magnetic field behaviour, where the field orientation is linked to the companion, was previously detected in HD 98088 and HD 161701, the two close main-sequence binaries with Ap components mentioned above. Obviously, a search for magnetic fields and the determination of their geometries in close binary systems is very important as the knowledge of the presence of a magnetic field and of the alignment of the mag- 
netic axis with respect to the orbital radius vector in Herbig binaries may hint at the mechanism of the magnetic field generation.

The weakness of the observed magnetic fields put into question the current understanding of the magnetospheric accretion process in intermediate-mass pre-main sequence stars. Importantly, Cauley \& Johns-Krull (2014) studied the He I $\lambda 10830$ morphology in a sample of 56 Herbig Ae/Be stars. They suggested that early Herbig Be stars do not accrete material from their inner disks in the same manner as T Tauri stars, while late Herbig Be and Herbig Ae stars show evidence for magnetospheric accretion. Furthermore, they proposed more compact magnetospheres in Herbig Ae/Be stars compared to T Tauri stars. Ababakr et al. (2017) presented $\mathrm{H} \alpha$ linear spectropolarimetry of a sample of $56 \mathrm{Herbig} \mathrm{Ae/Be} \mathrm{stars.} \mathrm{A} \mathrm{change} \mathrm{in} \mathrm{linear}$ polarization across this line was detected in $42(75 \%)$ objects, indicating that the circumstellar environment around these stars has an asymmetric structure on small spatial scales, which is typically identified with a disk. A second outcome of their research was the confirmation that Herbig Ae stars are similar to T Tauri stars in displaying a line polarization effect, while depolarization is more common among Herbig Be stars. Using near-infrared multi-epoch spectroscopic data obtained with the CRIRES and X-shooter spectrographs installed at the VLT, Schöller et al. (2016) examined the magnetospheric accretion in the Herbig Ae star HD 101412. Spectroscopic signatures in He I 10830 and Pa $\gamma$, two near-infrared lines that are formed in a Herbig star's accretion region, were presenting temporal modulation. The authors showed that this modulation is governed by the rotation period of this star and that the observed spectroscopic variability was explained within the magnetic geometry established earlier from magnetic field measurements by Hubrig et al. (2011).

To summarize, it appears that the as yet small number of magnetic Herbig Ae/Be stars can be due to both the weakness of the magnetic fields and the large measurement errors. According to Alecian (2014), the magnetic properties of Herbig Ae/Be stars must have been shaped at a very early phase of the stellar evolution. Using premain-sequence evolutionary tracks calculated with the CESAM code (Morel 1997), she concluded that even stars above three solar masses will undergo a purely convective phase before reaching the birthline. Therefore, it is reasonable to assume that the weak magnetic fields detected in a number of Herbig Ae/Be stars are just leftovers of the fields generated by pre-main-sequence dynamos during the convective phase. If this scenario is valid, we should expect a significantly larger number of Herbig stars possessing weak magnetic fields.

\section{Low-mass stars}

Magnetic fields play a key role in the evolution of solar-like low mass stars as they determine the angular momentum loss, shape the stellar wind, and produce highenergy electromagnetic and particle radiation. A solar mass star spends approximately $10 \mathrm{Gyr}$ on the main sequence. Over the main-sequence life, magnetic activity declines with age and is closely related to a loss of angular momentum (e.g. Sku- 
manich 1972). Long-term monitoring of stellar activity using chromospheric emission measured in the cores of $\mathrm{Ca}$ II $\mathrm{H} \& \mathrm{~K}$ lines revealed that the chromospheric flux increases with the rotation rate (Baliunas et al. 1995; Hall et al. 2007). However, not all active stars undergo smooth activity cycles and the activity of the most active stars has the tendency to fluctuate erratically, while regular activity cycles are observed in older stars. Egeland et al. (2017) studied the long-term variability of a sample of five solar analog stars using composite chromospheric activity records up to 50 years in length and synoptic visible-band photometry about 20 years long. Their sample covered a large range of stellar ages, which they used to represent the evolution of activity for solar mass stars. The authors found that young, fast rotators have an amplitude of variability many times higher than that of the solar cycle, while old, slow rotators have very little variability.

Typical aims in polarimetric studies of low-mass stars are to determine the topology of the surface magnetic field and to study the relation between magnetic fields and cool spots. Most recent studies apply the ZDI technique using Stokes $I V$ spectropolarimetry and it is not seldom that no correlation between cool spots and a detected magnetic field is found. Rosén et al. (2015) compared ZDI results using Stokes $I V$ and the full set of Stokes $I V Q U$ parameters for the evolved binary RS CVn-type variable II Peg. They found significant differences in the magnetic field solutions and underestimation of the total magnetic energy. The retrieval of the meridional field component especially benefited from using linear polarization.

Naturally, spectropolarimetric observations of low-mass stars provide excellent constraints for theoretical dynamo models. One would expect stars with an internal structure similar to that of the Sun to show the same type of dynamo operation. On the other hand, ZDI studies frequently find large spots at high latitudes and large regions of a near-surface azimuthal field (e.g. Järvinen et al. 2015). Petit et al. (2008) suggested that the magnetic field is mostly poloidal for low rotation rates and that more rapid rotators host a large-scale toroidal component in their surface field. Further, they inferred that a rotation period lower than about $12 \mathrm{~d}$ is necessary for the toroidal magnetic energy to dominate over the poloidal component. Metcalfe et al. (2016) proposed that a change in the character of differential rotation is the mechanism that ultimately disrupts the large-scale organization of magnetic fields in sun-like stars. The process begins at a Rossby number (associated with convective motions and presenting the ratio of the rotation period to the convective turnover time) close to one, where the rotation period becomes comparable to the convective turnover time. The Vaughan-Preston gap (Vaughan \& Preston 1980) can then be interpreted as a signature of rapid magnetic evolution triggered by a shift in the character of differential rotation, which is an emergent property of turbulent convection in the presence of Coriolis forces.

The red dwarfs are the coolest objects which include late $\mathrm{K}$ and $\mathrm{M}$ dwarfs. The majority of red dwarfs exhibit evidence of strong magnetic activity, which is expressed in strong optical flares, enhanced UV, X-ray, and radio emission. The process of generating strong magnetic fields in fully convective objects and those with very deep convection zones is not yet understood. First direct magnetic field measurements in these dwarfs were achieved using the Zeeman split Fe I line at $8468.4 \AA$ 
(Johns-Krull \& Valenti 1996). More recent spectropolarimetric observations demonstrated that early type $\mathrm{M}$ dwarfs have magnetic fields with a strong toroidal component, reminiscent of those of active $\mathrm{K}$ and $\mathrm{G}$ dwarfs, whereas the lowest-mass $\mathrm{M}$ dwarfs exhibit magnetic fields that are highly organized and strongly poloidal (e.g. Morin et al. 2008a) and some of them show indications of polar starspot features (Morin et al. 2008b).

Starspots are the primary evidence of magnetic activity harboring the strongest field and the coldest plasma. Spectropolarimetric surveys of red dwarfs revealed the presence of numerous atomic and molecular signatures formed in starspots and the chromosphere. First detections of polarization in $\mathrm{TiO}, \mathrm{CaH}$, and $\mathrm{FeH}$ transitions in starspots on M and K dwarfs were presented by Berdyugina et al. $(2006,2008)$ using ESPaDOnS. It was the first time that polarimetry of cool atmospheres with the help of molecular physics was used to obtain new insights into the magnetic structures of cool atmospheres. For the red dwarf AU Mic, the authors identified four spots, one of positive and three of negative polarity. These spots are 500-700 K cooler than the photosphere and harbour a maximum magnetic field of $5.3 \mathrm{kG}$.

Brown dwarfs, often referred to as "failed stars" because they do not sustain hydrogen fusion in their core, show magnetic activity similar to that of low-mass stars and produce huge flares (Paudel et al. 2018). The first direct detection of a strong, $5 \mathrm{kG}$ magnetic field on the surface of an active brown dwarf was presented in the work of Berdyugina et al. (2017). The surface magnetic field of the brown dwarf with a mass of about $55 M_{\mathrm{J}}$ and at an age of $22 \mathrm{Myr}$ was detected in circularly polarized signatures in the $819 \mathrm{~nm}$ sodium line. According to the authors, the young age of the magnetic brown dwarf implies that it may still maintain a disk, which may facilitate bursts via magnetospheric accretion, like in higher-mass T Tauri stars.

The study of magnetic fields of low-mass stars became particularly important after the discovery of exoplanets around such stars. Multiwavelength observations with different observing strategies concluded that a giant exoplanet in a short-period orbit can induce activity in the photosphere and upper atmosphere of its parent star. This makes the host star's magnetic activity a probe of the planet's magnetic field. The large scale magnetic field of the planet-host star HD 189733 with a mainly toroidal surface magnetic field with a strength of $20-40 \mathrm{G}$ has been observed over multiple years. The ZDI analysis of these observations revealed a structural evolution of the field between observations (e.g. Fares et al. 2013).

\section{Post-main-sequence stars}

The evolved binary components in RS CVn-type variables show remarkable activity, displaying strong chromospheric plages, coronal $\mathrm{X}$-ray and microwave emission and flares detected in the optical, UV, radio, and X-rays. For these systems, magnetic activity cycles have been suggested to be the cause of the detected period modulations. One of the RS CVn systems exhibiting substantial period variations is HR 1099 with a high level of magnetic activity in all available activity indicators. 
Studies using photometric observations reported different magnetic activity periods, from $14.1 \mathrm{yr}$ (Muneer et al. 2010) to $19.5 \mathrm{yr}$ (Lanza et al. 2006). The Applegate mechanism proposed by Applegate (1992) has recently been discussed by Völschow et al. (2018) as a potential intrinsic mechanism to explain transit timing variations in various kinds of close binary systems. In the framework of the Applegate model, period modulations can be explained by gravitational coupling of the orbit to variations in the shape of the magnetically active star in the system. The variable deformation of the active star is produced by variations in the distribution of angular momentum as the star goes through its activity cycle. The torque needed to redistribute the angular momentum can be exerted by a mean subsurface magnetic field of several kilogauss.

In former studies, an exciting topic of the magnetic activity of RS CVn-type variables and the FK Comae stellar group was the flip-flop phenomenon. The FK Comae stellar group consists of single G-K giants showing high activity and rapid rotation. In many such active stars, the spots were found to concentrate on two permanent active longitudes, which were half a period apart (see e.g. Berdyugina et al. 1998). In some of these stars, the dominant part of the spot activity concentrated on one of the active longitudes, and abruptly switched the longitude every few years. This flipflop phenomenon was discovered in FK Comae (Jetsu et al. 1993). Light curves and Doppler imaging showed evidence of differential rotation of modest strength. However, in his most recent work, Jetsu (2018) showed that the period search methods used in previous studies do not necessarily produce correct rotation period estimates, if there are multiple interfering periodicities. Based on his new two-dimensional period finding method, Jetsu argued that the observed deceptive light curves were the interference of two real constant period light curves of long-lived starspots. The slow motion of these long-lived starspots with respect to each other caused the observed light curve changes.

Hubrig et al. (1994) carried out magnetic field measurements for thirteen bright G-K giants of different X-ray luminosity and concluded that magnetic fields in such objects are indeed observable using spectropolarimetry. Later on, direct detections of magnetic fields in the photospheres of cool, evolved giants advancing on the red giant branch and the asymptotic giant branch were presented by KonstantinovaAntova et al. (2013) and Aurière et al. (2015). In the sample of 48 red giants studied by Aurière et al., magnetic fields were detected via Zeeman signatures in 29 giants. The majority of the magnetic giants are either in the first dredge up phase or at the beginning of core He burning, i.e. in phases when the convective turnover time is at a maximum. Four giants for which the magnetic field was measured to be outstandingly strong, up to $100 \mathrm{G}$ (with respect to that expected from the relation between rotation period and magnetic field or from their evolutionary status), were interpreted as being probable descendants of magnetic Ap stars.

One of the major open questions regarding the formation of planetary nebulae (PNe) concerns the mechanism that is responsible for their non-spherical, often axisymmetric shaping (e.g. Balick \& Frank 2002). Both, central star binarity and stellar rotation in combination with magnetic fields, are among the favourite explanations. Basically, the origin of PNe is understood to be a consequence of the 

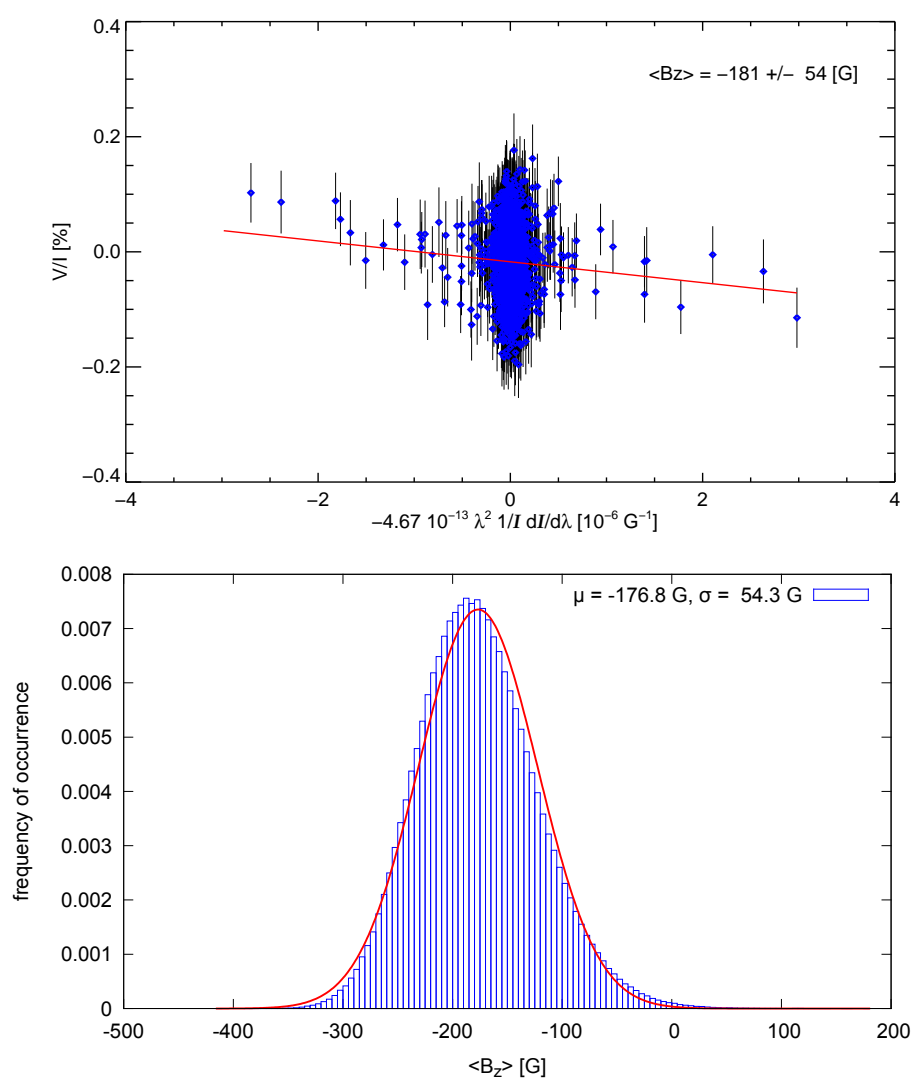

Fig. 3 IC 418: Regression detection of a $\left\langle B_{\mathrm{z}}\right\rangle=-181 \pm 54 \mathrm{G}$ mean longitudinal magnetic field, using all lines (top). The corresponding $\left\langle B_{\mathrm{Z}}\right\rangle$ distribution (bottom) deviates slightly from a Gaussian (red curve) and indicates $\left\langle B_{\mathrm{Z}}\right\rangle=-177 \pm 54 \mathrm{G}$. Figure from Steffen et al. (2014, A\&A, 570, A88); reproduced with permission, (C) ESO.

interaction of the hot central star with its circumstellar environment through photoionization and wind-wind collision. In principle, the role of magnetic fields in shaping PNe may be verified or disproved by empirical evidence, as already suggested by Jordan et al. (2005). Using FORS 1 in spectropolarimetric mode, they reported the detection of magnetic fields of the order of $\mathrm{kG}$ in the central stars of the PNe NGC 1360 and LSS 1362. A reanalysis of their data, however, did not provide any significant evidence for longitudinal magnetic fields that are stronger than a few hundred Gauss in these stars (Jordan et al. 2012). Their field measurements had typical error bars of 150 to $300 \mathrm{G}$. Similar results were achieved in the work of Leone et al. (2011), who concluded that the mean longitudinal magnetic fields in NGC 1360 and LSS 1362 are much weaker, less than $600 \mathrm{G}$, or that the magnetic field has a 
complex structure. The most recent search for magnetic fields in central stars of planetary nebulae by Leone et al. (2014), based on spectropolarimetric observations of 19 central stars with the Intermediate-dispersion Spectrograph and Imaging System (ISIS) on the William Herschel Telescope (WHT) and VLT/FORS 2, was partly affected by large measurement uncertainties and reported no positive detection either.

Using low-resolution polarimetric spectra obtained with FORS 2, Steffen et al. (2014) carried out a search for magnetic fields in a sample of 12 central stars covering the whole range of morphologies from round to elliptical/axisymmetric, and bipolar PNe, and including both chemically normal and Wolf-Rayet type (hydrogenpoor) central stars. The sample included two round nebulae (NGC 246, Hen 2-108), five elliptical nebulae (IC 418, NGC 1514, NGC 2392, NGC 3132, Hen 2-131), and three bipolar nebulae (NGC 2346, Hen 2-36, Hen 2-113). Two targets have WR-type central stars (NGC 246, Hen 2-113). In addition, the authors included the two (elliptical) targets of Jordan et al. (2005), NGC 1360 and LSS 1362, for which they originally claimed the detection of $\mathrm{kG}$ magnetic fields. Six of the 12 central stars were known binaries.

Formal $3 \sigma$ detections were achieved for IC $418\left(\left\langle B_{\mathrm{Z}}\right\rangle=-181 \pm 54 \mathrm{G}\right)$, for the WR-type central star Hen 2-113 $\left(\left\langle B_{\mathrm{z}}\right\rangle=-58 \pm 18 \mathrm{G}\right)$, and the weak emission line star Hen 2-131 $\left(\left\langle B_{\mathrm{Z}}\right\rangle=-120 \pm 32 \mathrm{G}\right)$. The mean longitudinal magnetic field $\left\langle B_{\mathrm{Z}}\right\rangle$ of the central stars was derived by linear regression employing two different methods: a) using a weighted linear regression line through the measured data points and b) generating $M=10^{6}$ statistical variations of the original dataset by the bootstrapping technique and analyzing the resulting distribution $P\left(\left\langle B_{\mathrm{Z}}\right\rangle\right)$ of the $M$ regression results. Mean and standard deviation of this distribution are identified with the most likely mean longitudinal magnetic field and its $1 \sigma$ error, respectively. The main advantage of this method is that it provides an independent error estimate. The results of both methods for the central star of the young, elliptical planetary nebula IC 418 are presented in Fig. 3. A mean longitudinal magnetic field of negative polarity was detected at a $3 \sigma$ significance level when using the entire spectrum: $\left\langle B_{\mathrm{Z}}\right\rangle=-181 \pm 54 \mathrm{G}$ using the first method and $\left\langle B_{\mathrm{Z}}\right\rangle=-177 \pm 54 \mathrm{G}$ for the second method. Most stars in the sample of Steffen et al. (2014) were not studied at the achieved accuracy before, permitting to put constraints on the strength of the magnetic fields in the central stars of planetary nebulae.

\section{Degenerate stellar remnants}

White dwarfs represent the final stage of stellar evolution for the majority of stars with masses less than about $8 M_{\odot}$. The majority of white dwarfs have hydrogenrich atmospheres (DA). Other types of white dwarfs include stars with atmospheres dominated by helium (DB) and those without a visual helium spectrum (DC). 25\% to $30 \%$ of white dwarfs show traces of heavy elements, i.e. elements heavier than 
helium (Zuckerman et al. 2003, 2010), probably due to accreted planetary or asteroidal debris (Jura 2008).

A significant fraction of white dwarfs possess a magnetic field ranging from a few $\mathrm{kG}$ up to several hundred MG (e.g. Ferrario et al. 2015). According to Kepler et al. (2013), the incidence of magnetism within the white dwarf population remains uncertain with an incidence of magnetism of about $5 \%$ in magnitude limited surveys, or between $10-20 \%$ in volume limited surveys (e.g. Kawka et al. 2007). Kawka \& Vennes (2014) showed that cool, polluted white dwarfs have a higher incidence of magnetism, up to $40 \%$, than in the general population of white dwarfs. Dufour et al. (2013) reported on a magnetic field incidence of about $70 \%$ in the rare class of warm and hot DQ white dwarfs, which present rare carbon-dominated objects known as hot DQ white dwarfs.

To study magnetic fields in white dwarfs, again circular polarization observations are involved. Similar to Ap and Bp stars, the geometry of the magnetic field in white dwarfs is assumed to be a centred or offset dipole. However, the magnetic field structure in white dwarfs studied over their rotational period shows a large diversity, from a simple offset dipole, over structures with spots, to multipoles (e.g. Kawka et al. 2018). For non-variable magnetic white dwarfs it is assumed that they are either rotating with a very long period or that their magnetic fields are nearly aligned with the rotation axis. A recent statistical analysis of the linear polarization properties between magnetic and non-magnetic white dwarfs showed no difference in the polarization degrees (Żejmo et al. 2017; Słowikowska et al. 2018). Such an analysis allowed the authors to select a set of good candidates of faint linear polarimetric standard stars that can be used as stable polarimetric calibration sources.

The mechanism responsible for the presence of magnetic fields in these stars remains unclear, as white dwarfs do not contain any significant convective zones. In the framework of the fossil field theory for the origin of magnetic fields, and assuming magnetic flux conservation, Ap and Bp stars are unlikely progenitors of white dwarfs with weak magnetic fields. Tout et al. (2008) proposed a binary origin, where the magnetic field is formed via a dynamo created during a common envelope phase. Nordhaus et al. (2011) proposed that during a CE phase a low-mass star will be tidally disrupted by its proto-white dwarf companion, forming an accretion disk and generating a dynamo in the disk, which is then transferred to the degenerate core via accretion. García-Berro (2012) suggested that magnetic fields can also be produced by the merger of two white dwarfs. To explain the sharp increase in the incidence of magnetism in cool polluted white dwarfs, Kawka \& Vennes (2014) argued that the phenomenon coincides with the likely presence of a perennially crowded circumstellar environment, which may have been caused by the same event that generated the magnetic field. As various classes of white dwarfs show a different incidence of magnetic fields, it is plausible that magnetic white dwarfs are created by several discernible processes.

For a discussion of the magnetic properties of neutron stars see Chapter 6. 


\section{References}

1. Ababakr, K.M., Oudmaijer, R.D., Vink, J.S.: 2017, MNRAS 472, 854

2. Abt, H.A., Conti, P.S., Deutsch, A.J., Wallerstein, G.: 1968, ApJ 153, 177

3. Adams, F.C., Gregory, S.G.: 2012, ApJ 744, 55

4. Alecian, E., et al.: 2009, MNRAS 400, 354

5. Alecian, E., et al.: 2013, A\&A 549, L8

6. Alecian, E.: 2014, in: Putting A Stars into Context: Evolution, Environment, and Related Stars, eds. G. Mathys, E.R. Griffin, O. Kochukhov, R. Monier, G.M. Wahlgren, p. 84

7. Applegate, J.H.: 1992, ApJ 385, 621

8. Auriére, M., et al.: 2015, A\&A 574, A90

9. Babcock, H.W.: 1947, ApJ 105, 105

10. Babcock, H.W.: 1958, ApJ 128, 228

11. Babcock, H.W.: 1960, ApJ 132, 521

12. Babel, J., Montmerle, T.: 1997, A\&A 323, 121

13. Bagnulo, S., Szeifert, T., Wade, G.A., Landstreet, J.D., Mathys, G.: 2001, Msngr. 104, 32

14. Balick, B., Frank, A.: 2002, ARA\&A 40, 439

15. Baliunas, S.L., et al.: 1995, ApJ 438, 269

16. Balona, L.A.: 2013, MNRAS 431, 2240

17. Balona, L.A.: 2016, MNRAS 457, 3724

18. Basri, G., Marcy, G.W., Valenti, J.A.: 1992, ApJ 390, 622

19. Berdyugina, S.V., Jankov, S., Ilyin, I., Tuominen, I., Fekel, F.C.: 1998, A\&A 334, 863

20. Berdyugina, S.V., Fluri, D., Petit, P., Fluri, D.M., Afram, N., Arnaud, J.: 2006, in Solar Polarization 4, ed. by R. Casini \& B.W. Lites, ASP Conf. Ser. 358, 381

21. Berdyugina, S.V., et al.: 2008, in 14th Cambridge Workshop on Cool Stars, Stellar Systems, and the Sun, ed. by G. van Belle, ASP Conf. Ser. 384, 175

22. Berdyugina, S.V., et al.: 2017, ApJ 847, 61

23. Borra, E.F., Deschatelets, D.: 2015, AJ 150, 146.

24. Braithwaite, J., Spruit, H.C.: 2004, Nature 431, 819

25. Cantiello, M., et al.: 2009, A\&A 499, 279

26. Cantiello, M., Braithwaite, J.: 2011, A\&A 534, A140

27. Carrier, F., North, P., Udry, S., Babel, J.: 2002, A\&A 394, 151

28. Carroll, T.A., Strassmeier, K.G., Rice, J.B., Künstler, A.: 2012, A\&A 548, A95

29. Cassinelli, J.P., Ignace, A., Bjorkman, J.E.: 1995, IAUS 163, 191

30. Castro, N., et al.: 2015, A\&A 581, A81

31. Cauley, P.W., Johns-Krull, C.M.: 2014, ApJ 797, 112

32. Chabrier, G., Küker, M.: 2006, A\&A 446, 1027

33. de la Chevrotiére, A., St-Louis, N., Moffat, A.F.J., MiMeS Collaboration: 2014, ApJ 781, 73

34. Donati, J.-F., et al.: 1997, MNRAS 291, 658

35. Donati, J.-F., et al.: 2015, MNRAS 453, 3706

36. Duchêne, G.: 2015, Astrophys. \& Space Sci. 355, 291

37. Dufour, P., Vornanen, T., Bergeron, P., Fontaine, A.B.: 2013, 18th European White Dwarf Workshop, ASP Conf. Ser. 469, 167

38. Egeland, R., Soon, W., Baliunas, S., Hall, J.C., Henry, G.W.: 2017, IAUS 328, 329

39. Fares, R., et al.: 2013, MNRAS 435, 1451

40. Ferrario, L., de Martino, D., Gänsicke, B.T.: 2015, Space Sci. Rev. 191, 111

41. Fossati, L., et al.: 2015, A\&A 574, A20

42. Friend, D.B., MacGregor, K.B.: 1984, ApJ 282, 591

43. Gaensler, B.M., et al.: 2005, ApJ 620, L95

44. García-Berro, E., et al.: 2012, ApJ 749, 25

45. Gregory, S.G., et al.: 2012, ApJ 755, 97

46. Grunhut, J.H., et al.: 2017, MNRAS 465, 2432

47. Hale, G.E.: 1908, ApJ 28, 315

48. Hall, J.C., Lockwood, G.W., Skiff, B.A.: 2007, AJ 133, 862 
49. Herbst, W., Eislöffel, J., Mundt, R., Scholz, A.: 2007, Protostars and Planets V, eds. B. Reipurth, D. Jewitt, \& K. Keil, University of Arizona Press, Tucson, p. 297

50. Hubrig, S., Plachinda, S.I., Hunsch, M., Schröder, K.-P.: 1994, A\&A 291, 890

51. Hubrig, S., North, P., Mathys, G.: 2000, ApJ 539, 352

52. Hubrig, S., North, P., Schöller, M.: 2005, AIPC 784, 145

53. Hubrig, S., North, P., Schöller, M.: 2007, Astr. Nachr. 328, 475

54. Hubrig, S., et al.: 2009, A\&A 502, 283

55. Hubrig, S., et al.: 2010, Astr. Nachr. 331, 361

56. Hubrig, S., et al.: 2011, A\&A 525, L4

57. Hubrig, S., et al.: 2014a, A\&A 564, L10

58. Hubrig, S., et al.: 2014b, MNRAS 440, L6

59. Hubrig, S., Carroll, T.A., Schöller, M., Ilyin, I.: 2015, MNRAS 449, L118

60. Hubrig, S., et al.: 2016, MNRAS 458, 3381

61. Hubrig, S., et al.: 2017, MNRAS 472, 400

62. Hubrig, S., et al.: 2018a, MNRAS 477, 3791

63. Hubrig, S., Schöller, M., Järvinen, S.P., Küker, M., Kholtygin, A.F., Steinbrunner, P.: 2018b, Astr. Nachr. 339, 72

64. Hunger, K., Groote, D.: 1999, A\&A 351, 554

65. Järvinen, S.P., et al.: 2015, A\&A 574, A25

66. Järvinen, S.P., et al.: 2018, ApJL 858, L18

67. Jetsu, L., Pelt, J., Tuominen, I.: 1993, A\&A 278, 449

68. Jetsu, L.: 2018, MNRAS, submitted, arXiv: 1808.02221

69. Johns-Krull, C.M., Valenti, J.A.: 1996, ApJ 459, L95

70. Johnstone, C.P., Jardine, M., Gregory, S.G., Donati, J.-F., Hussain, G.: 2014, MNRAS 437, 3202

71. Jordan, S., Werner, K., O’Toole, S.J.: 2005, A\&A 432, 273

72. Jordan, S., Bagnulo, S., Werner, K., O’Toole, S.J.: 2012, A\&A 542, A64

73. Jura, M.: 2008, AJ 135, 1785

74. Kawka, A., Vennes, S., Schmidt, G.D., Wickramasinghe, D.T., Koch, R.: 2007, ApJ 654, 499

75. Kawka, A., Vennes, S.: 2014, MNRAS 439, L90

76. Kawka, A., Vennes, S., Ferrario, L., Paunzen, E.: 2018, MNRAS, accepted, tmp2960

77. Kepler, S.O., et al.: 2013, MNRAS 429, 2934

78. Kitchatinov, L.L., Rüdiger, G.: 1999, A\&A 344, 911

79. Kochukhov, O., Shultz, M., Neiner, C.: 2019, A\&A 621, A47

80. Konstantinova-Antova, R., et al.: 2013, Bulgarian Astron. J. 19, 14

81. Küker, M., Rüdiger, G.: 1999, A\&A 346, 922

82. Küker, M., Stix, M.: 2001, A\&A 366, 668

83. Küker, M.: 2009, AIPC 1094, 77

84. Landolfi, M., Landi DeglÍnnocenti, E., Landi DeglÍnnocenti, M., Leroy, J. L.: 1993, A\&A 272,285

85. Lanza, A.F.: 2006, MNRAS 369, 1773

86. Leone, F., Martínez González, M.J., Corradi, R.L.M., Privitera, G., Manso Sainz, R.: 2011, ApJ 731, L33

87. Leone, F., Corradi, R.L.M., Martínez González, M.J., Asensio Ramos, A., Manso Sainz, R.: 2014, A\&A 563, A43

88. Leroy, J. L.: 1989, A\&A 215, 360

89. Mathys, G.: 1990, A\&A 232, 151

90. Mathys, G.: 1993, ASPC 44, 232

91. Mathys, G., Hubrig, S.: 2006, A\&A 463, 699

92. Mathys, G.: 2017, A\&A 601, A14

93. McKee, C.F., Ostriker, E.C.: 2007, ARA\&A 45, 565

94. Metcalfe, T.S., Egeland, R., van Saders, J.: 2016, ApJL 826, L2

95. Michaud, G.: 1970, ApJ 160, 641

96. Mohanty, S., Shu, F.H.: 2008, ApJ 687, 1323

97. Morel, P.: 1997, A\&AS 124, 597 
98. Morel, T., et al.: 2015, IAUS 307, 342

99. Morin, J., et al.: 2008a, MNRAS 390, 56

100. Morin, J., et al.: 2008b, MNRAS 384, 77

101. Moss, D.: 2003, A\&A 403, 693

102. Muneer, S., Jayakumar, K., Rosario, M.J., Raveendran, A.V., Mekkaden, M.V.: 2010, A\&A 521, A36

103. Nordhaus, J., Wellons, S., Spiegel, D.S., Metzger, B.D., Blackman, E.G.: 2011, Proc. Natl. Acad. Sci. 108, 3135

104. Pallavicini, R., et al.: 1981, ApJ 248, 279

105. Paudel, R.R., et al.: 2018, ApJ 861, 76

106. Petit, P., et al.: 2008, MNRAS 388, 80

107. Petrovic, J., Langer, N., van der Hucht, K.A.: 2005, A\&A 435, 1013

108. Pevtsov, A.A., et al.: 2003, ApJ 598, 1387

109. Preston, G.W.: 1969, ApJ 156, 967

110. Preston, G.W.: 1971, ApJ 164, 309

111. Robrade, J., Schmitt, J.H.M.M.: 2009, A\&A 497, 511

112. Rosén, L., Kochukhov, O., Wade, G.A.: 2015, ApJ 805, 169

113. Sana, H., et al.: 2012, Science 337, 444

114. Schmitt, D.: 1987, A\&A 174, 281

115. Schöller, M., et al.: 2016, A\&A 592, A50

116. Schöller, M., et al.: 2017, A\&A 599, A66

117. Semel, M.: 1989, A\&A 225, 456

118. Sennhauser, C., Berdyugina, S.V., Fluri, D.M.: 2009, ASPC 405, 543

119. Skumanich, A.: 1972, ApJ 171, 565

120. Słowikowska, A., Krzeszowski, K., Żejmo, M., Blinov, D., Reig, P.: 2018, MNRAS 479.5312

121. Steffen, M., et al.: 2014, A\&A 570, A88

122. Stello, D., et al.: 2016, PASA 33, 11

123. Sudnik, N.P., Henrichs, H.F.: 2016, A\&A 594, A56

124. Tout, C.A., Wickramasinghe, D.T., Liebert, J., Ferrario, L., Pringle, J.E.: 2008, MNRAS 387, 897

125. ud-Doula, A., Owocki, S.P.: 2002, ApJ 576, 413

126. ud-Doula, A., Owocki, S.P., Townsend, R.H.D.: 2008, MNRAS 385, 97

127. Uzdensky, D.A., MacFadyen, A.I.: 2006, ApJ 647, 1192

128. Vaughan, A.H., Preston, G.W.: 1980, PASP 92, 385

129. Vink, J.S., Drew, J.E., Harries, T.J., Oudmaijer, R.D.: 2002, ASPC 267, 439

130. Völschow, M., Schleicher, D.R.G., Banerjee, R., Schmitt, J.H.M.M.: 2018, A\&A 620, A42

131. Wade, G.A., et al.: 2011, ASPC 449, 262

132. Wade, G.A., et al.: 2012, MNRAS 425, 1278

133. Walborn, N.R.: 1972, AJ 77, 312

134. Weber, E.J., Davis, L.Jr.: 1967, ApJ 148, 217

135. Wheelwright, H.E., Oudmaijer, R.D., Goodwin, S.P.: 2010, MNRAS 401, 1199

136. Zaire, B., Guerrero, G., Kosovichev, A.G., Smolarkiewicz, P.K., Landin, N.R.: 2017, IAUS 328,30

137. Żejmo, M., Słowikowska, A., Krzeszowski, K., Reig, P., Blinov, D.: 2017, MNRAS 464,1294

138. Zuckerman, B., Koester, D., Reid, I.N., Hünsch, M.: 2003, ApJ 596, 477

139. Zuckerman, B., Melis, C., Klein, B., Koester, D., Jura, M.: 2010, ApJ 722, 725 